\title{
Interventions for treating fractures of the distal femur in adults
}

Amy Drahota, Yvette Revell-Smith

\section{Introduction}

This summary is based on a Cochrane review (Griffin, Parsons, Zbaeda, \& McArthur, 2015), that explores how to treat distal femur fractures in adults. Distal femur fractures are most commonly seen as a fall-related injury in older adults, but can also stem from high-energy impacts in younger people, and in people who have previously undergone total knee replacement (Elsoe, Ceccotti, \& Larsen, 2018).

Fractures may be treated conservatively, by immobilisation involving casts, braces, splints, or traction, or surgically with various systems involving screws, plates, frames, rods, or prostheses (von Keudell, Shoji, Nasr, Lucas, Dolan, \& Weaver, 2016). Methods of surgical fixation have become increasingly sophisticated, for example, with options of 'locking' plates (Steinberg, Elis, Steinberg, Salai, \& Ben-Tov, 2017). The implications of these treatment options are important for nursing care, since they may affect the patient's functional outcomes, length of stay, and potential for adverse effects (Moloney, Pan, Van Eck, Patel, \& Tarkin, 2016). Nurses are ideally placed to explain the various options to patients, as part of informed and shared decisionmaking, to improve patient outcomes.

\section{- Objective/s:}

The aim of this review was to determine the benefits and harms of various treatment options for adults with distal femur fractures.

The authors hoped to compare: (1) different types of conservative treatments; (2) surgical and non-surgical treatments; (3) different types of surgical methods. 


\section{- Intervention/Methods:}

The review authors included randomised and quasi-randomised controlled trials, comparing treatment options for distal femur fractures in adults. Trials solely focussed on children were excluded. Iatrogenic fractures were excluded, however acute periprosthetic fractures were included.

The review authors were interested in comparing any conservative or surgical treatment options (excluding pharmacological interventions), in the short, intermediate, and long term. The primary outcomes were patient-reported functional outcomes and adverse events.

Five databases (up to August/September 2014), a clinical trials registry (January 2015), conference proceedings (2006-2013), and reference lists of included studies, were searched. Study selection, data extraction, and risk of bias assessments were conducted in duplicate by independent review authors, with disagreements resolved through discussion. Treatment effects were calculated using risk ratios or mean differences, and 95\% confidence intervals, with fixed effects meta-analysis, where appropriate.

\section{Results:}

Seven small studies at high risk of bias, with 444 adults were included in the review. One study compared dynamic condylar screw (DCS) fixation to skeletal traction; treatments had similar risks of death, re-operation, and repeat procedures. There was a non-significant increased risk of pressure sores (0/20 versus 4/20) with skeletal traction, associated with prolonged immobilisation. The remaining studies compared surgical interventions: retrograde intramedullary nail (RIMN) fixation versus DCS (N=3), RIMN versus non-locking plate fixation $(\mathrm{N}=1)$, locking plate fixation versus single fixed-angle device $(\mathrm{N}=1)$, and RIMN versus locking plate fixation $(\mathrm{N}=1)$. Only the final comparison reported musculoskeletal function (non-significant), and there were no statistically significant differences in adverse events. 


\section{- Conclusions:}

The review authors conclude that the evidence is of very low quality, incomplete, and insufficient to inform practice. These conclusions appear fair, with respect to basing treatment decisions on available evidence. The current evidence suggests there is no significant difference between treatment options with regards to adverse events and functional outcomes. However these studies were small and of low quality, they did not always report on the important outcomes, and were likely to be insufficiently powered to detect any potential real differences that may exist. That aside, studies did highlight that the various treatment options are prone to adverse effects, and nurses should be mindful of these.

\section{- Implications for Practice:}

Innovations in the treatment of distal femur fractures are advancing at a far greater pace than the research evidence is delivering. Still, nurses should be mindful of areas in which they can improve patient outcomes, for example by helping to prevent the development of pressure sores associated with prolonged immobilisation. Nurses need to be fully informed of adverse effects so they can adopt a patient-centred approach and undertake a holistic patient assessment (e.g. considering environmental, physical, and social factors; do they live alone? Are they mobile? What impact do their conditions have on their life?). In this way the nurse, together with the patient, can share the decision making process, the patient can be fully informed, and concerns can be discussed, so that a tailor-made plan of care can be developed to meet the needs of each individual patient (Thompson \& Dowding, 2009, p221). The patient and their needs, should be the centre of care, not the condition or set treatment.

\section{- References:}

Griffin XL, Parsons N, Zbaeda MM, McArthur J. Interventions for treating fractures of the distal femur in adults. Cochrane Database of Systematic Reviews 2015, Issue 8. Art. No.: CD010606. DOI: 10.1002/14651858.CD010606.pub2. 
Elsoe, R., Ceccotti, AA., \& Larsen, P. (2018) Population-based epidemiology and incidence of distal femur fractures. International Orthopaedics. 42(1); 191-6. DOI: 10.1007/s00264-017-3665-1

von Keudell, A., Shoji, K., Nasr, M., Lucas, R., Dolan, R., \& Weaver, M.J. (2016) Treatment Options for Distal Femur Fractures. Journal of Orthopaedic Trauma. 30; S25-S27. doi: 10.1097/BOT.0000000000000621

Moloney, G.B., Pan, T., Van Eck, C.F., Patel, D., \& Tarkin, I. (2016) Geriatric distal femur fracture: Are we underestimating the rate of local and systemic complications? Injury. 47(8); 1732-6. DOI: 10.1016/j.injury.2016.05.024

Steinberg, E.L., Elis, J., Steinberg, Y., Salai, M., Ben-Tov, T., (2017) A doubleplating approach to distal femur fracture: A clinical study. Injury. 48(10); 2260-5. DOI: 10.1016/j.injury.2017.07.025

Thompson, C., \& Dowding, D. (2009) Essential Decision Making and Clinical Judgement for Nurses. Edinburgh: Churchill Livingstone, Elsevier. 